\title{
LINC00565 promotes the progression of colorectal cancer by upregulating EZH2
}

\author{
XIAXIA SHAO *, TAO ZHAO* ${ }^{*}$, LEI XI, YUHONG ZHANG, JIA HE, JIE ZENG and LICHUN DENG \\ Department of Oncology, The Affiliated Jiangyin Hospital of Southeast University \\ Medical College, Jiangyin, Jiangsu 214400, P.R. China
}

Received May 4, 2020; Accepted October 19, 2020

DOI: $10.3892 / \mathrm{ol} .2020 .12314$

\begin{abstract}
The present study aimed to illustrate the role of LINC00565 in aggravating colorectal cancer (CRC) by targeting enhancer of zeste homolog 2 (EZH2). The relative levels of LINC00565 and EZH2 in CRC tissues, based on their Tumor-Node-Metastasis stage and tumor size, were detected by reverse transcription-quantitative polymerase chain reaction. The diagnostic value of LINC00565 in CRC was assessed by depicting receiver operating characteristic curves. Pearson's correlation test was applied to analyze the expression correlation between LINC00565 and EZH2 in CRC tissues. The transfection efficacy of three LINC00565 small interfering RNAs was examined in CRC HCT116 and SW480 cell lines. After knockdown of LINC00565, the proliferative and migratory abilities of CRC cells were detected by Cell Counting Kit-8 and Transwell assays, respectively. The subcellular distribution of LINC00565 was analyzed, and the interaction between LINC00565 and EZH2 was determined by RNA immunoprecipitation. Finally, co-regulation of LINC00565 and $\mathrm{EZH} 2$ on $\mathrm{CRC}$ cell functions was explored by performing rescue experiments. Results showed that LINC00565 was upregulated in CRC tissues, especially in patients with stage III+IV and in those with large tumor sizes, suggesting its diagnostic value in CRC. EZH2 was also upregulated in CRC tissues, showing a positive correlation with LINC00565. LINC00565 was mainly expressed in the cytoplasm and was found to bind with EZH2. Validation was performed by overexpressing EZH2, which abolished the role of silenced LINC00565 in regulating proliferative and migratory abilities in CRC. Therefore, the upregulation of LINC00565 in
\end{abstract}

Correspondence to: Dr Lichun Deng, Department of Oncology, The Affiliated Jiangyin Hospital of Southeast University Medical College, 163 Shoushan Road, Jiangyin, Jiangsu 214400, P.R. China E-mail: 18921233265@163.com

*Contributed equally

Key words: LINC00565, enhancer of zeste homolog 2, colorectal cancer
CRC tissues was found to stimulate the aggravation of CRC by upregulating EZH2.

\section{Introduction}

Colorectal cancer (CRC) is a common malignant tumor of the digestive system that seriously endangers human lives (1). $\mathrm{CRC}$ is the second- and third-most common cancer in women and men, respectively. In 2012, 614,000 women (9.2\% of all new cancer cases) and 746,000 men (10.0\% of new cancer cases) were diagnosed with colorectal cancer worldwide (2). Incidence varies geographically, with the highest incidence in Australia and New Zealand, whereas Western Africa has the lowest incidence (2). In total, 40-50\% of patients with CRC develop tumor relapse, despite improvements in the diagnostic and therapeutic methods, leading to a poor prognosis (3). There is a need to clarify the molecular mechanisms underlying the occurrence and metastasis of CRC. Meanwhile, developing effective biomarkers and improving therapeutic strategies for $\mathrm{CRC}$ are the focuses of clinical research.

Long non-coding RNAs (lncRNAs) are transcripts longer than 200 nucleotides that cannot be translated into proteins (4); they are involved in the regulation of transcriptional, post-transcriptional and epigenetic cell events $(5,6)$. Growing evidence has identified that lncRNAs participate in various biological processes, including embryonic stem cells development, helper T-cell differentiation, autophagy, myocardial infarction, cellular senescence, apoptosis and metastasis of cancer cells, and chemotherapeutic resistance $(7,8)$. In addition, dysregulated lncRNAs are closely linked to tumor relapse and poor prognosis (9). To date, several lncRNAs have been revealed to be abnormally expressed in CRC profiling, such as CCAT1, HOTAIR and H19 (10-12). The present study aimed to identify novel lncRNAs that were dysregulated in the development of CRC. To comprehensively characterize those aberrantly expressed lncRNAs, we analyzed CRC and normal tissue RNA sequencing data downloaded from The Cancer Genome Atlas (TCGA). In order to identify CRC-associated lncRNAs, the literature was searched to identify a novel lncRNA named LINC00565 has already been discovered in gastric cancer (13). LINC00565 spans 2,478 bp length and is located on chromosome 13, which is significantly upregulated in gastric cancer (GC) tissues (13). LINC00565 drives the progression of ovarian 
cancer by targeting GAS6 (14). In addition, LINC00565 promotes proliferation and inhibits apoptosis of GC by targeting miR-665/AKT3 axis. however, its role in CRC is still unclear (13).

Enhancer of zeste homolog 2 (EZH2) is a histone methyltransferase that functions as the catalytic subunit of the polycomb repressive complex 2 (PRC2) (15). PRC2 methylates lysine 27 on histone 3 (H3K27), resulting in the transcriptional silencing of target genes. EZH2 is responsible for methylating lysine 27 of histone 3 to generate H3K27me3, a histone mark associated with a more condensed chromatin and transcriptional gene repression (15). A previous study suggested that silencing EZH2 can induce senescence of tumor cells, including breast cancer, ovarian cancer, prostate cancer and non-Hodgkin lymphoma (16). In CRC tissues, EZH2 is highly expressed and its expression level is known to be associated with the prognosis of CRC (17). The binding between EZH2 and lncRNAs triggers the specific binding of lncRNAs to proteins, thus regulating protein activity and function $(18,19)$. Recent studies have reported that IncRNAs recruit polycomb-group proteins to target genes $(18,19)$. A total of $20 \%$ of all human IncRNAs have been shown to physically associate with PRC2, suggesting that lncRNAs may have a general role in recruiting polycomb-group proteins to their target genes $(18,19)$. At present, to the best of our knowledge, no studies have shown whether LINC00565 can interact with EZH2 to influence the occurrence and development of CRC. Therefore, the present study aimed to explore the role of LINC00565 in the malignant progression of CRC and its interaction with EZH2, in order to provide new strategies for the clinical diagnosis and treatment of CRC.

\section{Patients and methods}

Samples. Samples of CRC were collected from 42 patients undergoing radical resection between May 2017 and May 2019 in The Affiliated Jiangyin Hospital of Southeast University Medical College (Jiangyin, China). There were 31 males and 11 females with an average age of (52.11 \pm 8.99$)$ years, and the patient age ranged from 37-75 years old. The inclusion criteria used were as follows: i) Diagnosis of patients based on the diagnostic criteria for CRC; and ii) Patients having had no prior CRC treatment. The exclusion criteria were as follows: i) Patients undergoing retreatment after cancer recurrence; and ii) Patients with other malignant tumors. The tumor node metastasis (TNM) stage of CRC was based on the American Joint Committee on Cancer CRC 7th edition TNM stage (20). Histology grade was determined based on a previous report (2). Normal tissues resected $\geq 5 \mathrm{~cm}$ away from the edge of CRC tissues were also collected, and these were pathologically confirmed to lack tumor invasive tissue. None of the recruited patients had undertaken CRC treatment. Intraoperatively collected tissue samples were immediately placed in liquid nitrogen and stored at $-80^{\circ} \mathrm{C}$ before use. The present study was approved by the Ethics Committee of The Affiliated Jiangyin Hospital of Southeast University Medical College (approval number JYH-17-03-11-932), and written informed consent was obtained from each subject. The clinical data for the patients is shown in Table SI.
Reverse transcription-quantitative polymerase chain reaction $(R T-q P C R)$. Total RNA was extracted from the tissues and cells using the TRIzol kit (Invitrogen; Thermo Fisher Scientific Inc.), respectively, followed by measurement of RNA concentration using an ultraviolet spectrophotometer (Hitachi Ltd.). The complementary Deoxyribose Nucleic Acid (cDNA) was synthesized according to the instructions of the PrimeScript ${ }^{\mathrm{TM}}$ RT MasterMix kit (Invitrogen; Thermo Fisher Scientific Inc.). qPCR was subsequently conducted following the recommendations of SYBR Premix Ex Taq ${ }^{\mathrm{TM}}$ (Takara Bio, Inc.). The following primer sequences were used for qPCR: GAPDH forward, 5'-CAC CCACTCCTCCACCTTTG-3' and reverse, 5'-CCACCA CCCTGTTGCTGTAG-3'; LINC00565 forward, 5'-GGC CTGAGCATTGCATAACG-3' and reverse, 5'-CTGATG GAGCGACCGTCTAC-3'; and EZH2 forward, 5'-TTGTTG GCGGAAGCGTGTAAAATC-3' and reverse, 5'-TCCCTA GTCCCGCGCAATGAGC-3'; and U6 forward, 5'-GCGCGT CGTGAAGCGTTC-3' and reverse, 5'-GTGCAGGGTCCG AGGT-3'. GAPDH was served as the internal control. The thermocycling conditions included pre-denaturation at $94^{\circ} \mathrm{C}$ for $5 \mathrm{~min}$, followed by 40 cycles of denaturation at $94^{\circ} \mathrm{C}$ for $30 \mathrm{sec}$, annealing at $55^{\circ} \mathrm{C}$ for $30 \mathrm{sec}$ and extension at $72^{\circ} \mathrm{C}$ for $90 \mathrm{sec}$. Gene expression levels were quantitatively analyzed using the $2^{-\Delta \Delta \mathrm{Cq}}$ method (21).

Cell culture. CRC cell lines (HCT116, SW480, HT-29 and LoVo) and the normal human colonic epithelial cell line (HCoEpiC) (cat. no. BSC-5005479458-01) were purchased from the American Type Culture Collection. Cells were cultured in Dulbecco's modified Eagle's medium (DMEM; Gibco; Thermo Fisher Scientific, Inc.) containing 10\% fetal bovine serum (FBS; Gibco; Thermo Fisher Scientific, Inc.) and $1 \%$ penicillin in a humidified incubator with $5 \% \mathrm{CO}_{2}$ at $37^{\circ} \mathrm{C}$. Medium was replaced every $24 \mathrm{~h}$. Cell passage was performed using $0.25 \%$ trypsin when cell density was $>70 \%$.

Cell transfection. CRC cell lines and HCoEpiC were resuspended in antibiotic-free DMEM and re-seeded into 6-well plates at a density of $3 \times 10^{5}$ cells/well. CRC cell lines and $\mathrm{HCoEpiC}$ were incubated for $18-24 \mathrm{~h}$ at $20^{\circ} \mathrm{C}$ and transfected using a Lipofectamine ${ }^{\circledR} 2000$ kit (Sigma-Aldrich; Merck KGaA). Cell transfection was performed at $20^{\circ} \mathrm{C}$ for $48 \mathrm{~h}$. Transfection efficiency was assessed by RT-qPCR. Liposomes, si-NC (empty vector), si-LINC00565 and pcDNA-EZH2 overexpression plasmid and pcDNA-NC (negative control) were all purchased from Shanghai Genechem Co. Ltd. Sequences of LINC00565 small interfering RNAs (siRNAs) were as follows: si-LINC00565 1, GCCTCCTGGTTTATAGCATGA; si-LINC00565 2, GGAGGACGCATCAATCCTTCT; and si-LINC00565 3, GCTTTGCGAAGACTTTCTTCT. siRNA at the concentration of $50 \mathrm{nM}$ was added to each well and then incubated for $48 \mathrm{~h}$ following which subsequent experimentation was performed.

Cell Counting Kit-8 (CCK-8) assay. Transfected CRC cell lines and HCoEpiC were cultured in 96-well plates at a concentration of $2 \times 10^{3}$ cells/well. Six replicates were set in two groups (si-NC and si-LINC00565). At 6, 24, 48, 72 and $96 \mathrm{~h}$, $10 \mu$ l CCK-8 solution (Dojindo Molecular Technologies, Inc.) 
was added per well and incubated for $4 \mathrm{~h}$. The CCK- 8 was used according to the manufacturer's protocol. Optical density at $450 \mathrm{~nm}$ was measured using a microplate reader.

Transwell migration assay. Transfected cells were prepared as a suspension of $1 \times 10^{9}$ cells $/ \mathrm{ml}$ in antibiotic-free DMEM. Next, $200 \mu \mathrm{l}$ of this cell suspension was added to the top of Transwell chambers (EMD Millipore), which were pre-inserted in a 24-well plate. In the bottom chambers, $600 \mu 1$ of antibiotic-free DMEM containing 10\% FBS was added. The difference in serum concentration was the inductive factor for cell chemotaxis. After incubation for $48 \mathrm{~h}$ at $37^{\circ} \mathrm{C}$, the invasive cells were fixed in $4 \%$ paraformaldehyde, dyed with crystal violet for 2 min at $37^{\circ} \mathrm{C}$ and counted using a light microscope (BX-42; Olympus Corporation). Penetrating cells were counted in ten randomly selected fields per sample (magnification, x10). Transwell migration assay was conducted following the same procedures except for Matrigel pre-coating (1 h at room temperature).

Chromatin fractionation. A total of $1 \times 10^{6}$ cells in the bottle were fully lysed in $200 \mu \mathrm{l}$ of lysis buffer (cat. no. QC25-05099; Qincheng Biotech Ltd.) and centrifuged at $4^{\circ} \mathrm{C}, 4,500 \times \mathrm{g}$ for 5 min to separate the cellular components. The supernatant contained the cytoplasmic fraction. Subsequently, the pellet was incubated in buffer SK and absolute ethanol. Nuclear RNAs were obtained by column (Invitrogen; Thermo Fisher Scientific, Inc.) centrifugation at $4^{\circ} \mathrm{C}, 4,500 \mathrm{x} g$ for $10 \mathrm{~min}$. Transfection efficiency was assessed by RT-qPCR. Liposomes, si-NC (empty vector), si-LINC00565 and pcDNA-EZH2 overexpression plasmid were all purchased from Shanghai Genechem Co. Ltd. Cytoplasmic and nuclear RNA levels were then determined by RT-qPCR.

RNA immunoprecipitation (RIP). CRC cell lines and HCoEpiC were treated following the manufacturer's instructions of Millipore Magna RIP RNA Binding Protein Immunoprecipitation kit (EMD Millipore). Cell debris was removed from $140 \mu \mathrm{l}$ of HepG2 2.2.15 supernatant or patient plasma by high-speed centrifugation $\left(20,800 \times \mathrm{g}\right.$ at $4^{\circ} \mathrm{C}$ for $5 \mathrm{~min})$. Samples were subsequently precleared by incubation with $50 \mu \mathrm{l}$ of Protein G MicroBeads (Protein G-Agarose; cat. no. SC-2003; Santa Cruz Biotechnology Inc.) at room temperature for $2 \mathrm{~h}$. The samples extracted $(10 \mu \mathrm{l})$ was used as input control. After application on a $\mu \mathrm{MACS}$ column, the flow-through pool was incubated with $2 \mu \mathrm{g}$ of rabbit monoclonal enhancer of zeste homolog 2 (EZH2) antibody (1:500; cat. no. ab191080; Abcam) or anti-IgG antibody $\left(1: 2,000\right.$; cat. no. ab6721; Abcam) at $4^{\circ} \mathrm{C}$ for $6 \mathrm{~h}$. Following application on a new $\mu$ MACS column and washing with phosphate-buffered saline, bead-bound fractions were eluted from the columns by using lysis buffer (Nanjing Jiancheng Bioengineering Institute) for RNA isolation. A protein RNA complex was captured and digested with $0.5 \mathrm{mg} / \mathrm{ml}$ proteinase $\mathrm{K}$ containing $0.1 \%$ SDS (Nanjing Jiancheng Bittech Company) to extract RNA. The magnetic beads were repeatedly washed with RIP washing buffer to remove non-specific adsorption as much as possible. Finally, the extracted RNA was subjected to mRNA level determination using RT-qPCR.
Statistical analysis. SPSS 19.0 software (IBM Corp.) was used for data analysis. Data were expressed as the mean \pm standard deviation. Each set of experiments was repeated three times. The diagnostic value of LINC00565 in CRC was assessed by depicting receiver operating characteristic (ROC) curves. Pearson's correlation test was conducted to analyze the correlation between LINC00565 and EZH2 expression. Comparison of pairwise differences between groups was performed using Student's t-test. Comparison between multiple groups was performed using one-way ANOVA followed by the post hoc Bonferroni test. $\mathrm{P}<0.05$ was considered to indicate a statistically significant difference.

\section{Results}

LINC00565 is upregulated in CRC. Firstly, the LINC00565 level in CRC tissues compared with normal tissues was assessed. As shown by the RT-qPCR results, LINC00565 was upregulated in CRC tissues compared with that in normal tissues (Fig. 1A). In addition, the classification of CRC tissues based on Tumor-Node-Metastasis (TNM) staging and tumor size, indicated that CRC tissues from more advanced TNM stages, such as stage III+IV, exhibited a higher level of LINC00565 than those from stage I+II tissues (Fig. 1B). Furthermore, CRC tissues with large tumor sizes $(>5 \mathrm{~cm})$ expressed a higher abundance of LINC00565 compared with those with small lesions (Fig. 1C). These results suggested that LINC00565 may be involved in the progression of CRC as an oncogene. To confirm the diagnostic value of LINC00565 in CRC, ROC curves were depicted to determine the area under the curve (AUC). The results of the ROC curves demonstrated that LINC00565 has diagnostic value in CRC (AUC, 0.8163; $\mathrm{P}<0.05$; cut-off value, 0.1236) (Fig. 1D).

LINC00565 promotes proliferative and migratory abilities in $C R C$. To investigate the effects of LINC00565 on proliferative and migratory abilities in CRC in vitro experiments were conducted to clarify the role of LINC00565 in regulating cell phenotypes of CRC. LINC00565 was highly expressed in CRC cell lines, especially SW480 and HCT116 cells, when compared with HCoEpiC control cells (Fig. 2A). In order to silence LINC00565 expression in SW480 and HCT116 cells, three siRNAs sequences were used. LINC00565 expression was inhibited by the transfection of all of the sequences (Fig. 2B and C), but si-LINC00565 3\# presented the best efficacy and was selected for the following experiments. Knockdown of LINC00565 markedly decreased proliferation in the HCT116 and SW480 cells (Fig. 2D and E). In addition, the migratory ability of the CRC cells was suppressed by the transfection of cells with si-LINC00565 3\# (Fig. 2F). These results suggested that knockdown of LINC00565 could statistically inhibit proliferative and migratory potentials in CRC.

LINC00565 positively regulates the expression levels of $E Z H 2$. Subsequently, whether EZH2 was involved in the progression of CRC was investigated. Subcellular distribution of LINC00565 was analyzed. LINC00565 was mainly expressed in the cytoplasm of HCT116 and SW480 cells (Fig. 3A and B). EZH2 is one member of PRC2 family (15). Compared with normal tissues, EZH2 expression levels were 

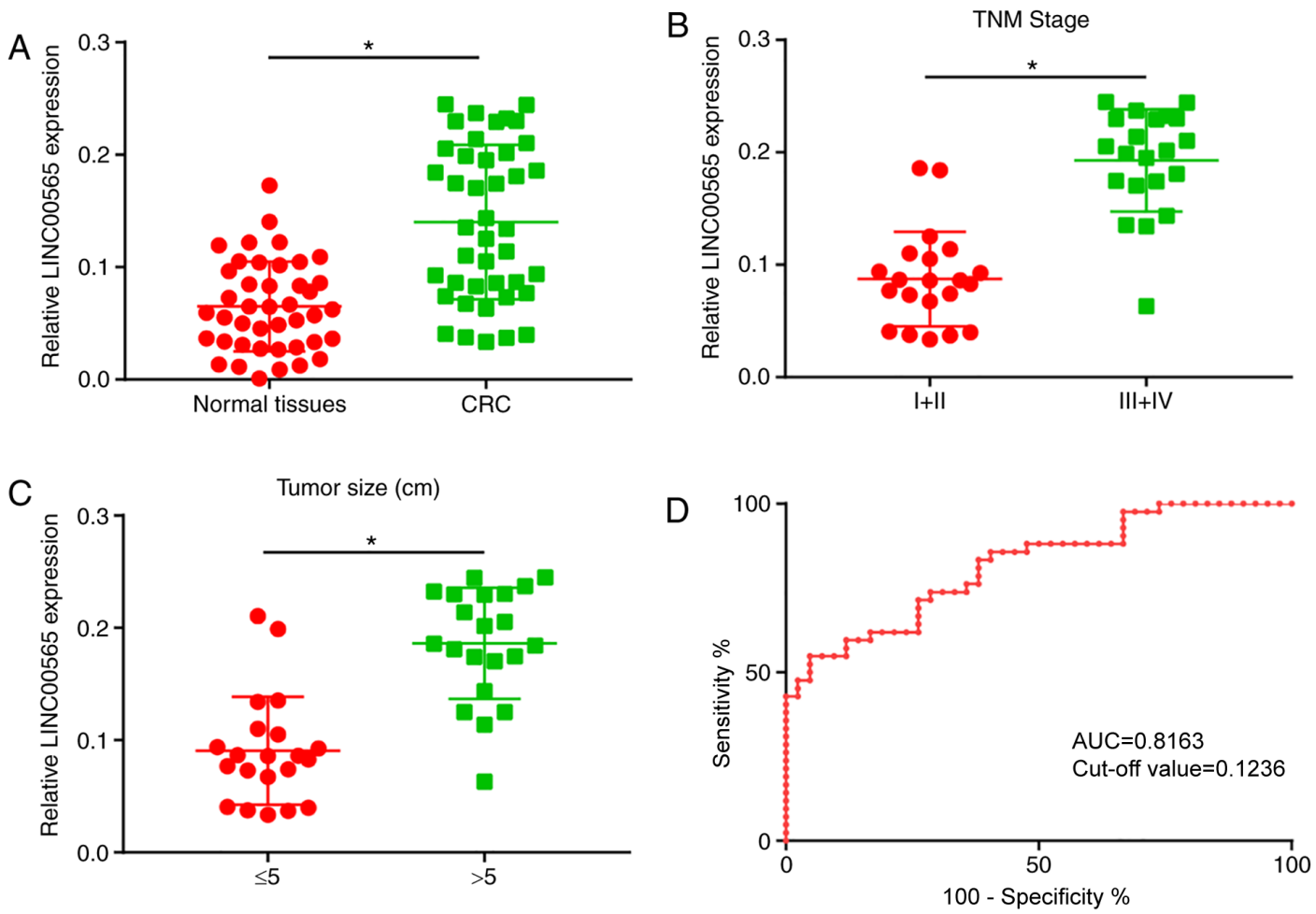

Figure 1. LINC00565 is upregulated in CRC. (A) Relative mRNA expression levels of LINC00565 in CRC tissues and normal tissues. (B) LINC00565 mRNA expression levels in CRC tissue samples from TNM stage I+II and stage III+IV. (C) LINC00565 mRNA expression levels in CRC tissues of tumors $\leq 5$ and $>5 \mathrm{~cm}$. (D) Receiver operating characteristic curve depicted based on the diagnostic value of LINC00565 in CRC (AUC, 0.8163; P<0.05; cut-off value, 0.1236). ${ }^{*} \mathrm{P}<0.05$. CRC, colorectal cancer; TNM, Tumor-Node-Metastasis; AUC, area under the curve.
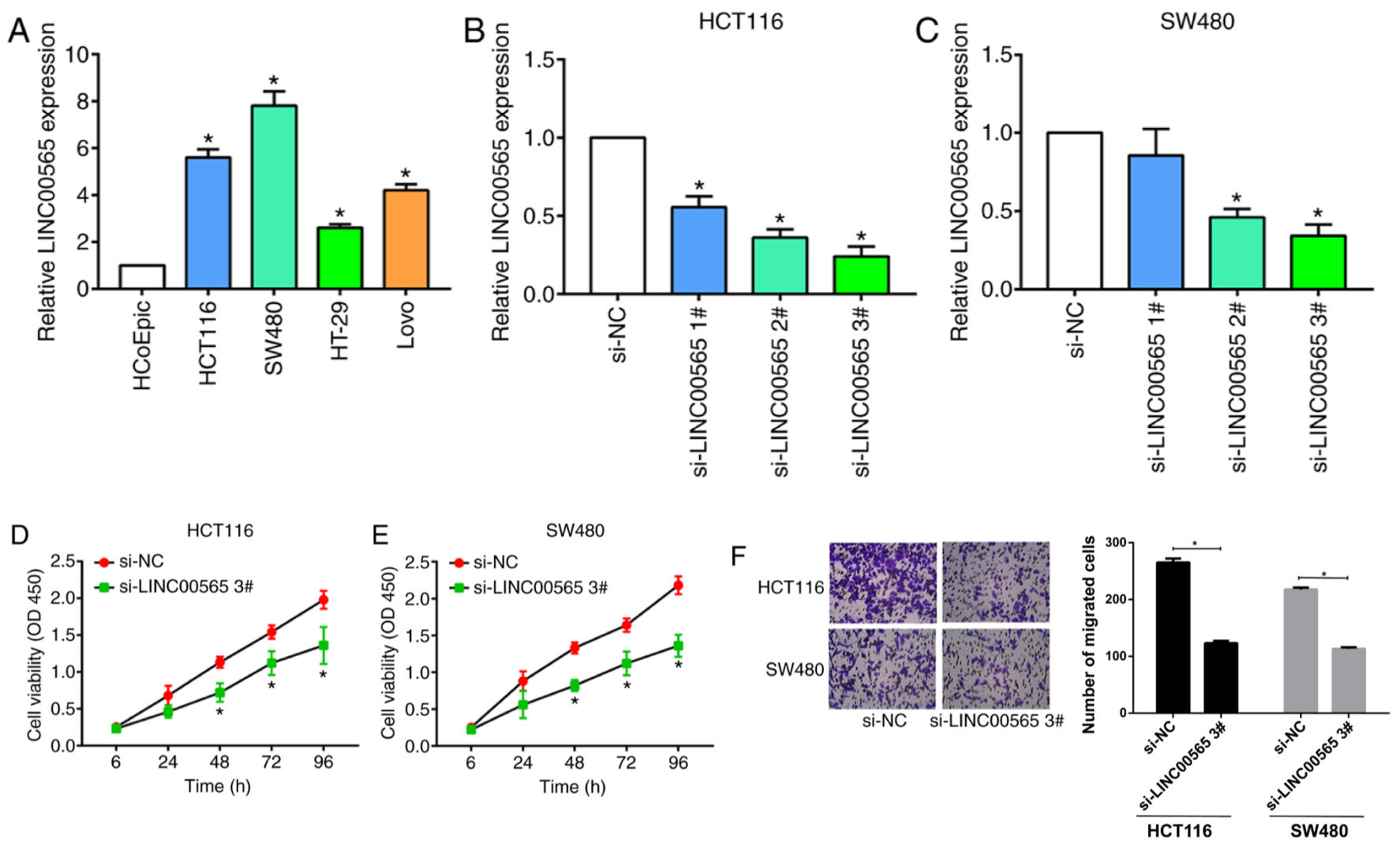

Figure 2. LINC00565 promotes proliferative and migratory abilities in CRC. (A) Relative mRNA expression levels of LINC00565 in CRC cell lines and the control HCoEpic cell line. "P<0.05 vs. HCoEpic. Transfection efficacy of si-LINC00565 1\#, si-LINC00565 2\# and si-LINC00565 3\# in (B) HCT116 and (C) SW480 cell lines. "P<0.05 vs. si-NC. Cell proliferation in (D) HCT116 and (E) SW480 cells transfected with si-NC or si-LINC00565 3\#. (F) Migration assay in HCT116 and SW480 cells transfected with si-NC or si-LINC00565 3\#. Magnification, x20. "P<0.05 vs. si-NC. CRC, colorectal cancer; si, small interfering RNA; NC, negative control; OD, optical density. 

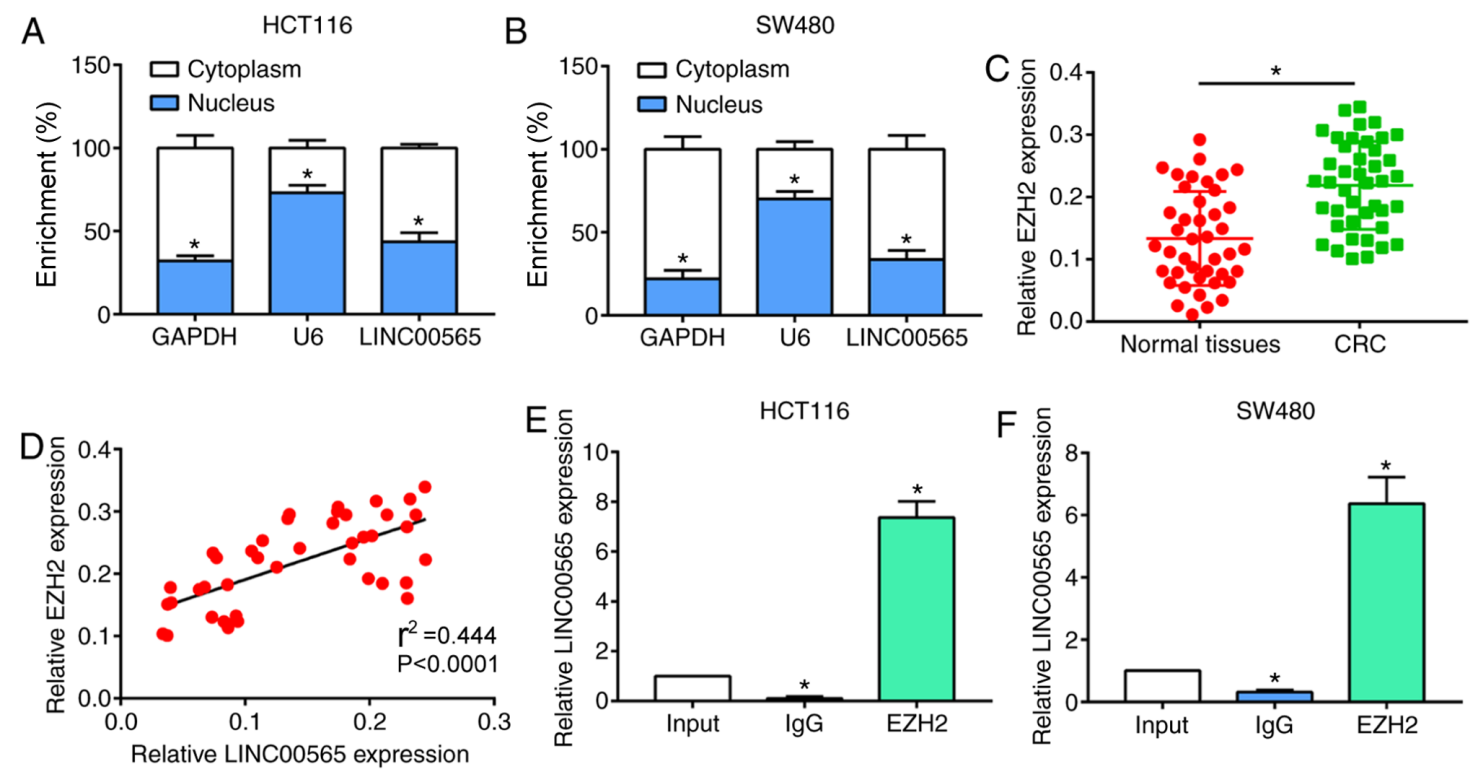

Figure 3. LINC00565 positively regulates the expression levels of EZH2. Cytoplasmic and nuclear distributions of LINC00565 in (A) HCT116 and (B) SW480 cell lines. "P<0.05 vs. Cytoplasm. (C) Relative mRNA expression levels of EZH2 in CRC tissues and normal tissues. "P<0.05 (D) Pearson's correlation analysis show a positive correlation between the mRNA expression levels of LINC00565 and EZH2 in CRC tissues. Immunoprecipitant of LINC00565 in input, anti-IgG and anti-EZH2 in (E) HCT116 and (F) SW480 cells. "P<0.05 vs. Input. EZH2, enhancer of zeste homolog 2; CRC, colorectal cancer.
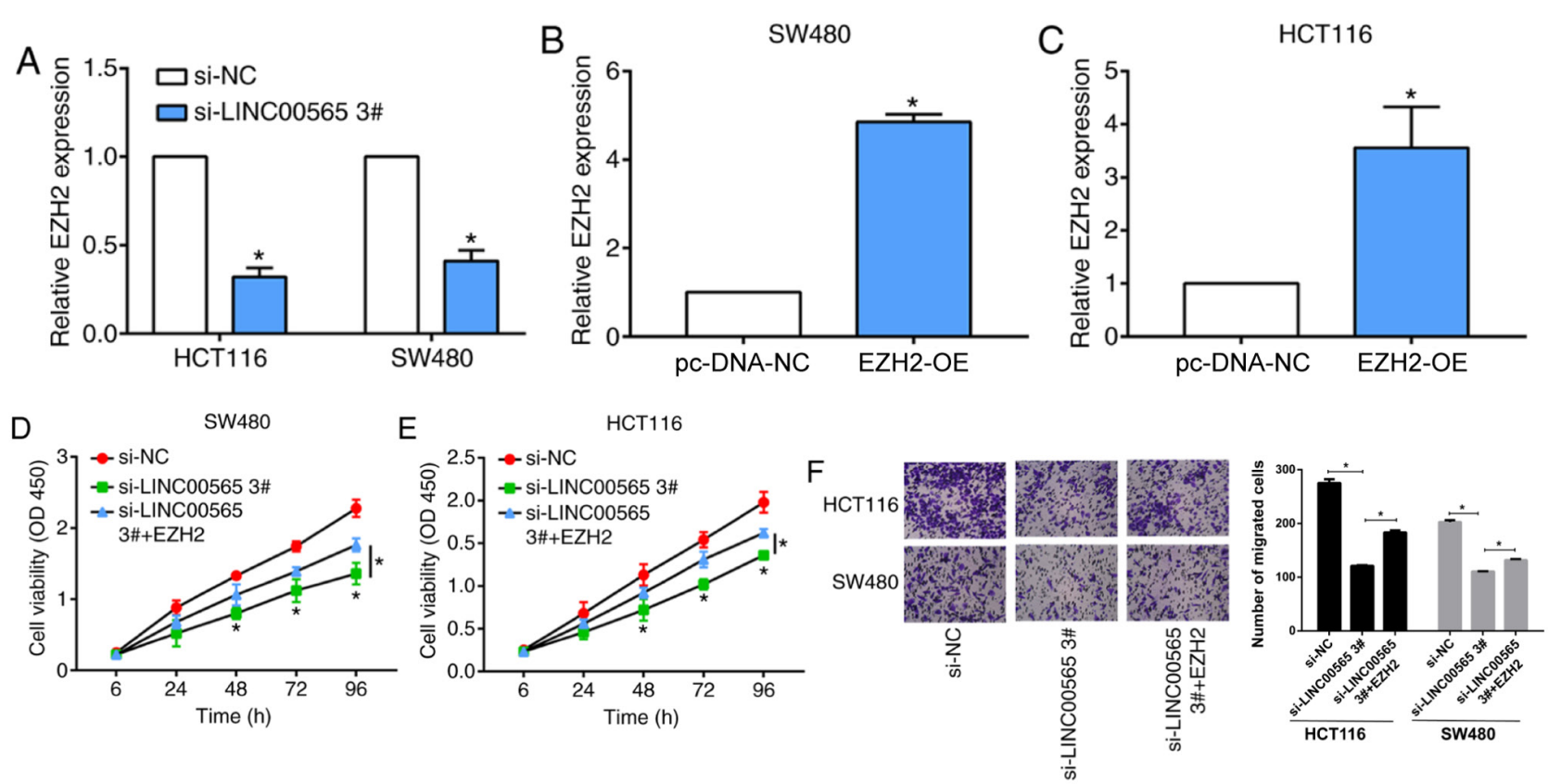

Figure 4. Effect of LINC00565 in colorectal cancer progression requires EZH2. (A) Relative mRNA EZH2 expression levels in HCT116 and SW480 cells transfected with si-NC or si-LINC00565 3\#. ${ }^{*} \mathrm{P}<0.05$ vs. si-NC. (EZH2 mRNA expression levels in (B) SW480 and (C) HCT116 cells transfected with pcDNA-NC or pcDNA-EZH2. ${ }^{*} \mathrm{P}<0.05$ vs. Control. Cell proliferation in (D) HCT116 and (E) SW480 cells transfected with si-NC, si-LINC00565 3\# or si-LINC00565 3\# + pcDNA-EZH2. (F) Migration assay in HCT116 and SW480 cells transfected with si-NC, si-LINC00565 3\# or si-LINC00565 3\# + pcDNA-EZH2. Magnification, x20. ${ }^{*} \mathrm{P}<0.05$. EZH2, enhancer of zeste homolog 2; si, small interfering RNA; NC, negative control.

upregulated in CRC tissues and were positively correlated with the expression levels of LINC00565 (Fig. 3C and D). This result suggested that EZH2 may also be involved in the progression of CRC. The interaction between LINC00565 and EZH 2 was determined by RIP. Furthermore, LINC00565 was found to be largely enriched in EZH2, indicating that there is an interaction between LINC00565 and EZH2 (Fig. 3E and F).
Effect of LINC00565 in CRC progression requires EZH2. Next, whether the effects of LINC00565 on CRC progression require EZH2 was studied. EZH2 was found to be downregulated upon transfection of si-LINC00565 3\# in CRC cells (Fig. 4A). Next, pcDNA-EZH2 overexpression plasmid was used to transfect cells, which resulted in a marked increase of EZH2 expression levels in SW480 and HCT116 cells (Fig. 4B and C). EZH2 overexpression was found to 
partially reverse the decreased proliferation observed with LINC00565-knockdown in CRC cells (Fig. 4D and E). In addition, the reduced migratory ability of CRC cells transfected with si-LINC00565 $3 \#$ was also attenuated by the overexpression of EZH2 (Fig. 4F). These results indicated that LINC00565 promoted proliferative and migratory potentials in $\mathrm{CRC}$ by positively regulating EZH2 expression levels.

\section{Discussion}

CRC is a prevalent tumor, ranking globally as the third and second most common tumor in men and women, respectively (2). In 2012, 1.4 million individuals were diagnosed with CRC, causing 693,900 deaths (22). According to the latest report, the incidence and mortality rates of $\mathrm{CRC}$ in the United States were in the top three among other cancers in 2012 (1). Great improvements have been made with regards to the diagnosis and treatment of CRC. However, the incidence of CRC in Asia still shows an upward trend (22). As a result, early diagnosis and the development of CRC biomarkers are of clinical significance.

IncRNAs were initially detected from DNA transcripts by Okazaki et al (23). Currently, a large number of tumor-associated lncRNAs have been identified. For example, Kogo et al (11) demonstrated that the lncRNA HOTAIR was upregulated in CRC tissues when compared with non-tumoral tissues. Highly expressed HOTAIR also predicted liver metastasis and a poor prognosis in CRC. KEGG pathway analysis revealed that HOTAIR induces gene silencing by interacting with the PRC2 family (including SUZ12, EZH2 and H3K27me3) and LSD1 (9). Thus, it is possible that CRC-associated lncRNAs can be utilized as diagnostic and therapeutic targets. However, the regulatory mechanisms of CRC-specific lncRNAs in cancer progression should be further validated. LINC000565 has been reported to be significantly increased in ovarian cancer and to be closely associated with the prognosis of ovarian cancer (14). Notably, in the present study, LINC00565 was found to play a similar role in CRC, with LINC00565 being upregulated in CRC tissues when compared with normal tissues, and higher levels of LINC00565 being detected in advanced stages of CRC and in those with larger tumor sizes. In addition, in vitro assays showed the promotive effects of LINC00565 on the proliferative and migratory potential of CRC cells. The results suggested that LINC00565 might be an oncogene involved in CRC progression.

$\mathrm{EZH} 2$ was also upregulated in CRC tissues in the present study. Pearson's correlation analysis showed a positive correlation between the mRNA expression levels of LINC00565 and EZH2. A number of studies have shown that EZH2 may serve a crucial role in the occurrence and development of CRC. In CRC tissues, EZH2 is highly expressed and its expression level is known to be associated with the prognosis of CRC (17). Xie et al (24) found that MALAT1 functioned as a competing endogenous RNA to promote CRC development and EZH2 expression through sponging miR-363-3p in vitro and in vivo. As a catalytic subunit of PRC2, EZH2 silences target genes by catalyzing $\mathrm{H} 3 \mathrm{~K} 27 \mathrm{me} 3$ and the methylation of lysine at position 9 of histone $\mathrm{H} 3$ through its highly conserved region in histone methyltransferase (15). Functionally, EZH2 regulates gene activities at the chromosome level, which are responsible for mediating cell growth, differentiation and other cellular functions (25). For example, 20\% of all human lncRNAs have been demonstrated to physically associate with EZH2, suggesting that lncRNAs may have a general role in recruiting polycomb-group proteins to their target genes $(18,19)$. Previous studies have reported that upregulated EZH2 in CRC samples is closely related to its degree of invasiveness, lymphatic metastasis and differentiation level. In addition, EZH2 is considered as an independent factor influencing the prognosis in CRC (26,27). In the present study, EZH2 was shown to interact with LINC00565. Importantly, it was able to attenuate the regulatory effects of LINC00565 on CRC cell phenotypes. Collectively, LINC00565 and EZH2 synergistically stimulated the aggravation of $\mathrm{CRC}$, indicating their potential as $\mathrm{CRC}$ biomarkers to monitor disease progression.

In summary, to the best of our knowledge, the present study is the first to find that LINC00565 is upregulated in CRC tissues, which stimulates the aggravation of CRC by upregulating EZH2. The current findings indicated that EZH2 was involved in the oncogenic role of LINC00565 in CRC. However, the specific mechanism of LINC00565 regulating $\mathrm{EZH} 2$ in the occurrence and development of CRC remains to be further studied.

\section{Acknowledgements}

Not applicable.

Funding

No funding was received.

\section{Availability of data and materials}

The datasets used and/or analyzed during the current study are available from the corresponding author on reasonable request.

\section{Authors' contributions}

XS, TZ, LX and LD designed the study and performed the experiments, JH, YZ and JZ analyzed the data, XS, LX, YZ, TZ and LD prepared the manuscript. All authors read and approved the final manuscript.

\section{Ethics approval and consent to participate}

This study was approved by the ethics committee of The Affiliated Jiangyin Hospital of Southeast University Medical College (approval. number. JYH-17-03-11-932). Written informed consent was obtained from the patients and/or guardians.

\section{Patients consent for publication}

Patients or their guardians have provided written informed consent for publication.

\section{Competing interests}

The authors declare that they have no competing interests. 


\section{References}

1. Siegel RL, Miller KD and Jemal A: Cancer Statistics, 2017. CA Cancer J Clin 67: 7-30, 2017.

2. Dekker E, Tanis PJ, Vleugels J, Kasi PM and Wallace MB: Colorectal cancer. Lancet 394: 1467-1480, 2019.

3. Weiser MR, Landmann RG, Kattan MW, Gonen M, Shia J, Chou J, Paty PB, Guillem JG, Temple LK, Schrag D, et al: Individualized prediction of colon cancer recurrence using a nomogram. J Clin Oncol 26: 380-385, 2008.

4. Mercer TR and Mattick JS: Structure and function of long noncoding RNAs in epigenetic regulation. Nat Struct Mol Biol 20: 300-307, 2013.

5. Deniz E and Erman B: Long noncoding RNA (lincRNA), a new paradigm in gene expression control. Funct Integr Genomics 17: 135-143, 2017.

6. Vance KW and Ponting CP: Transcriptional regulatory functions of nuclear long noncoding RNAs. Trends Genet 30: 348-355, 2014.

7. Huarte M: The emerging role of lncRNAs in cancer. Nat Med 21: $1253-1261,2015$.

8. Shi T, Gao G and Cao Y: Long noncoding RNAs as novel biomarkers have a promising future in cancer diagnostics. Dis Markers 2016: 9085195, 2016.

9. Yildirim E, Kirby JE, Brown DE, Mercier FE, Sadreyev RI Scadden DT and Lee JT: Xist RNA is a potent suppressor of hematologic cancer in mice. Cell 152: 727-742, 2013.

10. He X, Tan X, Wang X, Jin H, Liu L, Ma L, Yu H and Fan Z: C-Myc-activated long noncoding RNA CCAT1 promotes colon cancer cell proliferation and invasion. Tumour Biol 35 12181-12188, 2014

11. Kogo R, Shimamura T, Mimori K, Kawahara K, Imoto S, Sudo T, Tanaka F, Shibata K, Suzuki A, Komune S, et al: Long noncoding RNA HOTAIR regulates polycomb-dependent chromatin modification and is associated with poor prognosis in colorectal cancers. Cancer Res 71: 6320-6326, 2011.

12. Liang WC, Fu WM, Wong CW, Wang Y, Wang WM, Hu GX, Zhang L, Xiao LJ, Wan DC, Zhang JF and Waye MM: The lncRNA H19 promotes epithelial to mesenchymal transition by functioning as miRNA sponges in colorectal cancer. Oncotarget 6: 22513-22525, 2015.

13. Hu J, Ni G, Mao L, Xue X, Zhang J, Wu W, Zhang S, Zhao H, Ding L and Wang L: LINC00565 promotes proliferation and inhibits apoptosis of gastric cancer by targeting miR-665/AKT3 axis. Onco Targets Ther 12: 7865-7875, 2019.

14. Gong M, Luo C, Meng H, Li S, Nie S, Jiang Y, Wan Y, Li H and Cheng W: Upregulated LINC00565 accelerates ovarian cancer progression by targeting GAS6. Onco Targets Ther 12 : 10011-10022, 2019.

15. Li Z, Hou P, Fan D, Dong M, Ma M, Li H, Yao R, Li Y, Wang G, Geng P, et al: The degradation of EZH2 mediated by lncRNA ANCR attenuated the invasion and metastasis of breast cancer. Cell Death Differ 24: 59-71, 2017.
16. Tsang DP and Cheng AS: Epigenetic regulation of signaling pathways in cancer: Role of the histone methyltransferase EZH2. J Gastroenterol Hepatol 26: 19-27, 2011.

17. Bremer S,ConradiLC, Mechie NC,Amanzada A,Mavropoulou E, Kitz J, Ghadimi M, Ellenrieder V, Ströbel P, Hessmann E, et al: Enhancer of zeste homolog 2 in colorectal cancer development and progression. Digestion 6: 1-9, 2019.

18. Hirata H, Hinoda Y, Shahryari V, Deng G, Nakajima K, Tabatabai ZL, Ishii N and Dahiya R: Long noncoding RNA MALAT1 promotes aggressive renal cell carcinoma through Ezh2 and interacts with miR-205. Cancer Res 75: 1322-1331, 2015.

19. Cerase A, Smeets D, Tang YA, Gdula M, Kraus F, Spivakov M, Moindrot B, Leleu M, Tattermusch A, Demmerle J, et al: Spatial separation of xist RNA and polycomb proteins revealed by superresolution microscopy. Proc Natl Acad Sci USA 111: 2235-2240, 2014.

20. Hashiguchi Y,Hase K, Kotake K, Ueno H, Shinto E, Mochizuki H, Yamamoto J and Sugihara K: Evaluation of the seventh edition of the tumour, node, metastasis (TNM) classification for colon cancer in two nationwide registries of the United States and Japan. Colorectal Dis 14: 1065-1074, 2012.

21. Livak KJ and Schmittgen TD: Analysis of relative gene expression data using real-time quantitative PCR and the 2(-Delta Delta C(T)) method. Methods 25: 402-408, 2001.

22. Torre LA, Bray F, Siegel RL, Ferlay J, Lortet-Tieulent J and Jemal A: Global cancer statistics, 2012. CA Cancer J Clin 65: 87-108, 2015.

23. Okazaki Y, Furuno M, Kasukawa T, Adachi J, Bono H, Kondo S, Nikaido I, Osato N, Saito R, Suzuki H, et al: Analysis of the mouse transcriptome based on functional annotation of 60,770 full-length cDNAs. Nature 420: 563-573, 2002.

24. Xie JJ, Li WH, Li X, Ye W and Shao CF: LncRNA MALAT1 promotes colorectal cancer development by sponging miR-363-3p to regulate EZH2 expression. J Biol Regul Homeost Agents 33: 331-343, 2019.

25. Kondo Y, Shen L, Cheng AS, Ahmed S, Boumber Y, Charo C, Yamochi T, Urano T, Furukawa K, Kwabi-Addo B, et al: Gene silencing in cancer by histone $\mathrm{H} 3$ lysine 27 trimethylation independent of promoter DNA methylation. Nat Genet 40: 741-750, 2008.

26. Mimori K, Ogawa K, Okamoto M, Sudo T, Inoue H and Mori M: Clinical significance of enhancer of zeste homolog 2 expression in colorectal cancer cases. Eur J Surg Oncol 31: 376-380, 2005.

27. Wang CG, Ye YJ, Yuan J, Liu FF, Zhang H and Wang S: EZH 2 and STAT6 expression profiles are correlated with colorectal cancer stage and prognosis. World J Gastroenterol 16: 2421-2427, 2010.

This work is licensed under a Creative Commons Attribution-NonCommercial-NoDerivatives 4.0 International (CC BY-NC-ND 4.0) License. 